\title{
Numerical and Analytical Solutions of Hypersonic Interactions Involving Surface Property Discontinuities
}

\author{
Peter A. Gnoffo* \\ George R. Inger ${ }^{\dagger}$ \\ NASA Langley Research Center Iowa State University \\ Hampton, VA 23681-0001_ Ames, Iowa 50011
}

\begin{abstract}
The local viscous-inviscid interaction fleld generated by a wall temperature jump on a flat plate in supersonic flow and on the windside of a Reusable Launch Vehicle in hypersonic flow is studied in detail by both a Navier-Stokes numerical code and an analytical triple-deck model. Treatment of the rapid heat transfer changes both upstream and downstream of the jump is included. Closed form relationships derived from the triple-deck theory are presented. The analytically predicted pressure and heating variations including upstream influence are found to be in generally good agreement with the Computational Fluid Dynamic (CFD) predictions. These analyses not only clarify the interactive physics involved but also are useful in preliminary design of thermal protection systems and as an insertable module to improve CFD code efficiency when applied to such small-scale interaction problems. The analyses only require conditions at the wall and boundary-layer edge which are easily extracted from a baseline, constant wall temperature, CFD solution.
\end{abstract}

\section{Nomenclature}

$C$

$C_{p}$

$C_{1-6}$

$H$

$L$

$M$.

$n$

$p$

Pr

$q_{w}$

$R e_{L}$

$T$

V

$x, y, z$

$\tilde{x}, \tilde{y}$

$x_{J}$

$\beta$

$\delta$

$\epsilon$

$\Delta T_{w}$

$\kappa$

$\lambda$

$\mu$

$\omega$ $\rho \quad$ density, $\mathrm{kg} / \mathrm{m}^{3}$

\section{Subscripts}

$A D$ adiabatic wall conditions

$e \quad$ conditions at boundary layer edge

0 undisturbed boundary layer ahead of interaction zone

REF based on Reference Temperature

$\frac{T_{R E F}}{T_{e}}=.50+.039 M_{e}^{2}+.5 \frac{T_{w}}{T_{e}}$

$w \quad$ wall surface conditions

$\infty \quad$ freestream conditions

,$+-\quad$ conditions downstream and upstream of jump, respectively

Superscripts

() nondimensionalized by triple-deck theory

\section{Introduction}

Surface property discontinuities on a hypersonic vehicle are most often associated with the junction of two different thermal protection materials. They may also be associated with abrupt loss or deposition of surface coatings or changes in thermal conductivity and heat capacity of sensors on a test article. The abrupt jump in surface properties (catalytic efficiency, emissivity) in the presence of dissociated flow in hypersonic flight will lead to abrupt changes in heat transfer rate. The interactive thermophysics induced by such surface property jumps are remarkably similar to the phenomena induced by an artificially imposed temperature jump; consequently, insight into the catalytic jump problem may be gained through study of the temperature jump problem. ${ }^{1}$
- Aerothermodynamics Branch, Associate Fellow AIAA

†Professor, Dept. of Aerospace Engr. \& Engr. Mechanics, Associate Fellow AIAA

Copyright (C) 1999 by the American Institute of Aeronautics and Astronautics, Inc. No copyright is asserted in the United States under Title 17, U.S. Code. The U.S. Government has a royalty-free license to exercise all rights under the copyright claimed herein for governmental purposes. All other rights are reserved by the copyright owner. 
While the induced heating rate associated with discontimuous surface properties can be very large, the length scale of the interaction is usually poorly resolved by conventional computational fluid dynamic (CFD) grids. A purely numerical analysis of the problem can be addressed by a local grid refinement in the streamwise direction until the interaction is fully resolved. However, a better approach - one that provides physical insight and reduces the computer time required for design - is to couple a local, analytic solution of the interaction region around the discontinuity with a CFD solution obtained on a conventional grid.

This paper reviews the progress to date on the computational and analytical methods used to analyze the catalytic and temperature jump problems as presented in previous papers. ${ }^{1,2}$ The most recent analytical work on the temperature jump problem has revealed the necessity of going to a triple-deck analysis in order to pick up the important upstream interactions that were missed in a simpler integral-boundary-layer approach. ${ }^{1}$ Finally, it presents for the first time a test of the analytic, triple-deck analysis of problems of more general relevance to hypersonic, lifting bodies. The original triple-deck and computational analysis considered only a flat plate, for which analytic solutions in the transformed coordinates can be obtained. In the more general application, a computationally derived coordinate - one that is matched to the baseline CFD solution - will be used within the triple-deck analysis to match the physics in the interaction region. This approach is guided by a similar coupling procedure that was used with great success in problems with global changes in surface catalysis. ${ }^{3}$

The long range goal of this work is to provide analytic tools coupled with computational data sets to be used in the design of hypersonic vehicles in an Intelligent Synthesis Environment (ISE). Given a single, baseline CFD solution, the analytic tools allow a designer to investigate effects of continuous global changes or discontinuous local changes to thermal protection system surface properties on heating.

\section{Problem Definition}

As schematically illustrated in Fig. 1 we consider an incoming non-adiabatic laminar boundary layer with supersonic external flow that encounters an arbitrary wall temperature jump $\left(T_{w}^{-}\right.$to $\left.T_{w}^{+}\right)$at some arbitrary streamwise station $L$. The incoming boundary layer profile is modeled by self-similar compressible flat plate velocity and total enthalpy profiles, while the overlaying disturbed external inviscid flow is assumed to be governed by linearized supersonic theory. Furthermore, the Reynolds number $R e_{L}$ is taken to be very large such that the small parameter $\epsilon=R e_{L}^{-1 / 8}$ is small compared to unity along with the boundary-layer thickness ratio $\delta_{0} / L \sim \epsilon^{4}$. Finally, as confirmed by numerical results, ${ }^{1}$ the effects of streamwise diffusion

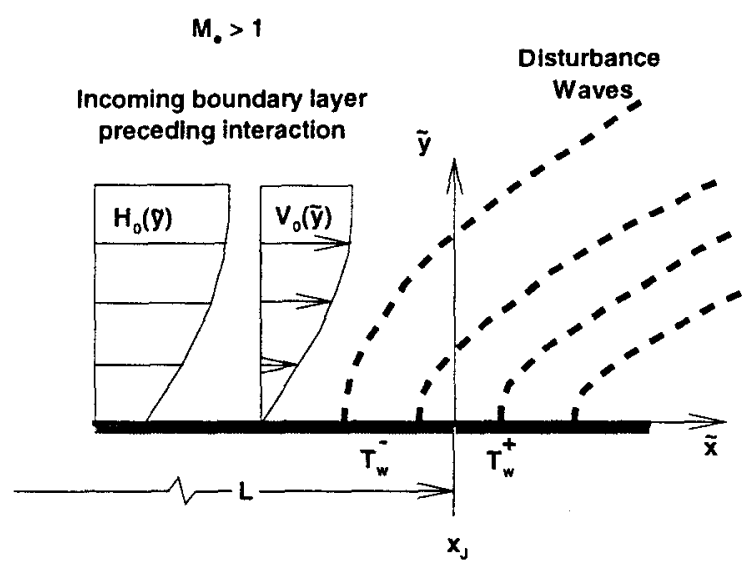

Fig. 1 Schematic of interaction generated by a wall temperature jump.

may be neglected even within the very short scale of the jump-generated interaction zone as long as the gas flow behaves as a continuum.

We note that this problem is phenomenologically similar to the case of an abrupt jump in surface catalytic efficiency. Such a case was originally studied ${ }^{4}$ using integral boundary-layer theory and compared to computational fluid dynamic (CFD) simulation. The results of that study are presented here in Fig. 2. Grid convergence of the heating rate across the discontinuity is evident in the CFD results designated by the symbols. Integral boundary-layer theory, given by the solid line in Fig. 2, assumes that the jump in heating and subsequent relaxation across the discontinuity may be predicted without consideration of upstream influence. Ignoring upstream influence leads to a large overestimate of the jump in heating rate and the relaxation distance to the asymptotic heating level. An ad-hoc correction for upstream influence in the integral boundary-layer theory, given by the dashed line, bounds the CFD results from below.

This result suggested that a triple-deck analysis which implicitly includes the upstream influence would be a more appropriate approach for problems involving discontinuous jumps in boundary conditions. A tripledeck analysis of a supersonic flow involving a temperature jump was chosen as a more tractable "pathfinder" before embarking on the more complex triple-deck analysis of a nonequilibrium, catalytic jump problem. The derivation of this triple-deck analysis is originally presented in an earlier paper. ${ }^{1}$ This paper focuses on the application of the theory to more general problems.

\section{Triple-Deck Formulation}

Details of the triple-deck formulation are available in an earlier paper ${ }^{1}$ and are not repeated here. Only the final analytic relations describing the upstream and 


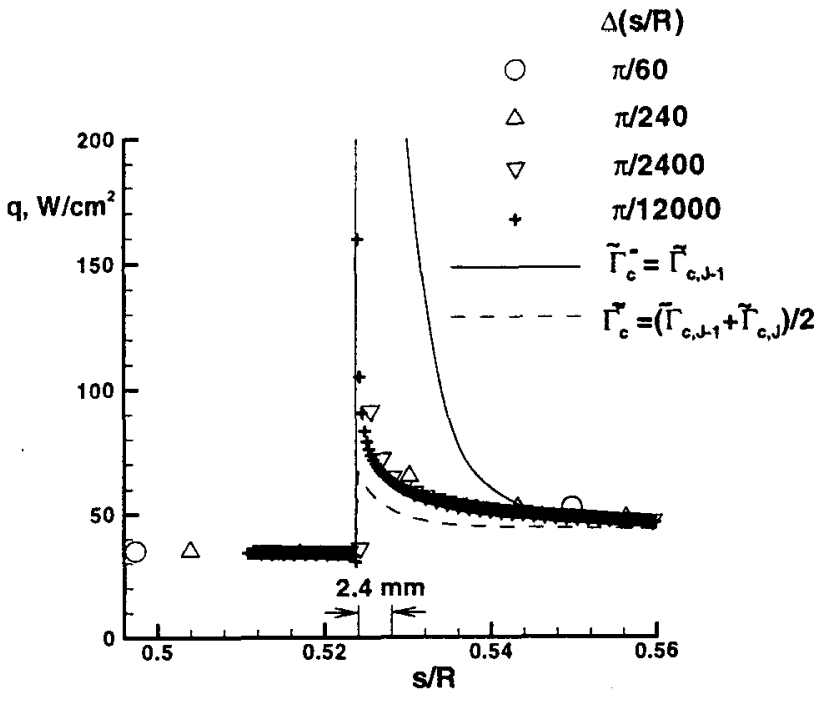

Fig. 2 Grid refinement study of convective heating on sphere in vicinity of discontinuous jump in surface catalycity and comparison to integral boundary-layer theory. (see Reference 4)

downstream solution for pressure and heating are presented. The appropriately non-dimensionalized variables with $\tilde{x}$ equal to 0 at the jump as noted in Fig. 1 are defined:

$$
\begin{gathered}
\hat{x} \equiv \frac{[\tilde{x} / L] \lambda^{5 / 4} \beta^{3 / 4}}{\epsilon^{3} C_{R E F}^{3 / 8}\left(T_{R E F} / T_{\infty}\right)^{3 / 2}\left(T_{w} / T_{R E F}\right)^{\omega+1 / 2}} \\
\hat{p} \equiv \frac{\left[\left(p-p_{\infty}\right) / \rho_{\infty} V_{\infty}^{2}\right] \beta^{1 / 2}}{\epsilon^{2} C_{R E F}^{1 / 4} \lambda^{1 / 2}} \\
\hat{q} \equiv \frac{q_{w}}{q_{w, 0}}
\end{gathered}
$$

The viscosity exponent $\omega$ is set to 0.75 herein.

Upstream Relations: $\hat{x}<0$

$$
\begin{aligned}
& \hat{p}(\hat{x})=C_{1}\left(\frac{\Delta T_{w}}{T_{w}}\right) e^{\kappa \hat{x}} \\
& \hat{q}=1-C_{2}\left(\frac{\Delta T_{w}}{T_{w}}\right) e^{\kappa \hat{x}}
\end{aligned}
$$

where

$$
\begin{gathered}
C_{1}=(3 / 4)(0.729) / P_{r}^{1 / 3} \kappa^{2 / 3} \\
C_{2}=(3 / 4)(0.315)\left[1+0.196\left(1-P_{r}\right)\right]
\end{gathered}
$$

and other referenced variables are defined in the Nomenclature.
Downstream Relations: $\hat{x}>0$

$$
\begin{aligned}
\hat{p}(\hat{x})= & C_{3}\left(\frac{\Delta T_{w}}{T_{w}}\right) \int_{0}^{\infty} \frac{\left(t+t^{-1 / 3}\right) e^{-\kappa t \hat{x}}}{t^{8 / 3}+t^{4 / 3}+1} d t \\
\hat{p}(\hat{x}) \simeq & C_{4}\left(\frac{\Delta T_{w}}{T_{w}}\right) \hat{x}^{-2 / 3} \quad \text { for large } \hat{x} \\
\hat{q}= & 1-\left\{\left(\frac{H_{0_{w}}}{H_{0_{A D}}-H_{0_{w}}}\right)\left[1+\frac{C_{5}}{\epsilon \hat{x}^{1 / 3}}\right]\right. \\
& \left.-C_{6} \int_{0}^{\infty} \frac{t^{1 / 3} e^{-\kappa t \hat{x}}}{t^{8 / 3}+t^{4 / 3}+1} d t\right\}\left(\frac{\Delta T_{w}}{T_{w}}\right)
\end{aligned}
$$

where

$$
\begin{gathered}
C_{3}=\sqrt{3}(0.729) / 2 \pi P_{r}^{1 / 3} \kappa^{2 / 3} \\
C_{4}=\sqrt{3}(0.729) \Gamma(2 / 3) / 2 \pi P_{r}^{1 / 3} \kappa^{4 / 3} \\
C_{5}=\frac{\sqrt{3}(0.729) \Gamma(1 / 3) \beta^{1 / 4}}{2 \pi \lambda^{1 / 4} C_{R E F}^{1 / 8}\left(T_{w} / T_{\infty}\right)^{1 / 2}} \\
C_{6}=\frac{4}{3} \sqrt{3} C_{2} / 2 \pi
\end{gathered}
$$

The integrals in Eqs. 8 and 10 are evaluated numerically.

\section{Coupling to CFD for General Applications}

For the flat plate test case, freestream conditions (subscript $(\infty)$ ) are nearly identical to boundary-layer edge conditions (subscript $(e)$ ). In more general applications we assume a locally two-dimensional flow over the domain in which the interaction occurs. In these cases edge conditions replace freestream conditions for all quantities used in Equations 1 - 14. Furthermore, the background reference pressure used in the definition of $\hat{p}$ in Eq. 2 and the reference heating heating level $q_{w, 0}$ used in Eq 3 are replaced by the locally varying value $p_{e}(x)$ and $q_{w}(x)$ from the baseline CFD solution. Use of these baseline reference values allows us to isolate the perturbations caused by the jump condition.

Also in the general case, the reference length $L$ at jump location $x_{J}$ required for the Reynolds number $R e_{L}$ is derived from the integral boundary-layer theory expression for the local, undisturbed value of $q_{w}$ in the baseline CFD solution. This approach is identical to the algorithm used to compute effects of global changes in surface catalytic efficiency on heating from a baseline CFD solution. ${ }^{3}$ Consequently, given a baseline CFD solution for the heating rate $q_{w}$ the reference length $L$ is implicitly computed from

$$
L \simeq V_{e} / \bar{\chi} \quad \text { for } 2-\mathrm{D} \text { flow }
$$


where

$$
\sqrt{\rho_{u} \mu_{w} \overline{\mathrm{X}}}=\frac{-P_{r} q_{w \cdot 0}}{Q_{w} C_{p} T_{e}} .
$$

The non-dimensional heating level $Q_{w}$, defined in Ref. 3, includes effects of nonequilibrium boundarylayer chemistry and surface catalytic efficiency.

\section{Test Cases}

\section{Flat Plate}

Test conditions for the flat plate are presented in Table 1. The plate is $30 \mathrm{~cm}$ long with a sharp leading edge radius of $0.1 \mathrm{~mm}$. The flow is laminar, steady, and two-dimensional at $\alpha=0$. The Mach 6 test case is chosen to be compatible with operating conditions in the LaRC 20 inch Mach 6 facility for possible future testing. The Mach 4 and Mach 2 test conditions with $R e_{L}=2.710^{6}$ match the total enthalpy of the Mach 6 test condition. The sensitivity of the results to Reynolds number was checked by introducing a variation in freestream density at Mach 2 and Mach 4. The lower Reynolds number also provides a better match to local test conditions for the RLV test case discussed in the next subsection. The high Reynolds number, Mach 2 case most closely matches the conditions assumed in the triple-deck analysis.

The wall temperature $T_{w}^{-}$is set to $300 \mathrm{~K}$. Three jumps in surface temperature acros $x_{J}$, corresponding to $\Delta T_{w} / T_{w}^{-}$equal to $-0.1,0.1$, and 0.2 are considered. Two values for $x_{J}$ equal to $5.06 \mathrm{~cm}$ and $20.455 \mathrm{~cm}$ were simulated in the Mach 6 case to quantify the influence of leading edge effects and boundary-layer growth; the pressure and heating relaxation phenomena being investigated here associated with the temperature jump occur on a comparable scale to these background influences. Only the results for the jump at $x_{J}=20.455$ $\mathrm{cm}$ are presented here, and values of $R e_{L}$ presented in Table 1 are based on this location. Comparisons of numerical simulation and triple deck theory at $x_{J}=5.06$ are qualitatively and quantitatively similar to the comparisons that follow at $x_{J}=20.455 \mathrm{~cm}$.

Table 1 - Flat Plate Test Conditions

\begin{tabular}{|c|c|c|c|c|c|}
\hline $\bar{M}_{\infty}$ & $R e_{L}$ & $\rho_{\infty}$ & $V_{\infty}$ & $T_{\infty}$ & $T_{w}$ \\
\hline 2 & $2.710^{6}$ & $3.43310^{-1}$ & 654.6 & 265.9 & 300. \\
2 & $2.710^{5}$ & $3.43310^{-2}$ & 654.6 & 265.9 & 300. \\
4 & $2.710^{6}$ & $1.26310^{-1}$ & 881.6 & 120.4 & 300. \\
4 & $2.710^{5}$ & $1.26310^{-2}$ & 881.6 & 120.4 & 300. \\
6 & $2.710^{6}$ & $5.85010^{-2}$ & 956.0 & 63.0 & 300. \\
\hline
\end{tabular}

Reusable Launch Vehicle (RLV) Test Case

The Reusable Launch Vehicle (RLV) is the B1001 Lockheed configuration defined in Phase I design. Only the windside forebody is considered in this test. The freestream test conditions, originally considered in the catalytic jump paper, ${ }^{3}$ are defined in Table 2. The flow simulation is at $\alpha=45 \mathrm{deg}$. and $79.6 \mathrm{~km}$ altitude. Surface temperature is held fixed at $900 \mathrm{~K}$ in the baseline CFD simulation. A five-species model for air and a fully catalytic wall boundary condition are applied.

A temperature jump of $90 \mathrm{~K}$ and $900 \mathrm{~K}\left(\Delta T_{w} / T_{w}=\right.$ 0.1 and 1.0 , repectively) is imposed at an axial location $25.3 \mathrm{~m}$ downstream of the nose. This location occurs near the peak of the recompression following the overexpansion of the flow from the nose along the centerline at $\alpha=45 \mathrm{deg}$. The imposed wall temperature of $900 \mathrm{~K}$ is the approximate value of the radiative equilibrium wall temperature in the vicinity of the jump. The parameter $\Delta T_{w} / T_{w}=1.0$ is not small in the context of triple-deck approximations; however, $\left(\Delta T_{w} / T_{w}\right)\left(T_{w} / T_{A D}\right)$ is small in this application and so the theory is tested in this range.

Two locations across the temperature jump, the first along the windside centerline and the second at an outboard location just inside of the chine, are studied. Boundary-layer edge conditions at each location are recorded in Table 2 . The equivalent length derived from the local baseline heating rate at these locations and integral boundary-layer theory as discussed previously is also recorded in Table 2 . It is this equivalent length that is used to define $R e_{L}$.

Table 2 - RLV Local Test Conditions

\begin{tabular}{|c|c|c|c|c|c|c|}
\hline Location & $M_{e}$ & $R e_{L}$ & $L$ & $\rho$ & $V$ & $T$ \\
\hline$\infty$ & 25.3 & $1.310^{5}$ & 15.5 & $1.910^{-5}$ & 7148. & 199. \\
$e, C L$ & 2.3 & $2.210^{5}$ & 24.9 & $2.310^{-4}$ & 4228. & 5709. \\
$e$, outboard & 2.6 & $8.710^{4}$ & 8.7 & $2.310^{-4}$ & 4562. & 5670. \\
\hline
\end{tabular}

\section{Code Description}

\section{Numerical Simulation}

The computational fluid dynamic (CFD) solutions are provided by the Langley Aerothermodynamic Upwind Relaxation Algorithm (LAURA). ${ }^{5-7}$ Comparisons to experimental data for hypersonic flows in air are documented in the literature. ${ }^{8-12}$ The code employs upwind-biased, point- and line-implicit relaxation. Inviscid fluxes are approximated with Roe's averaging, ${ }^{13}$ eigenvalue limiting (similar to Harten ${ }^{14}$ ), and Yee's symmetric total variation diminishing scheme. ${ }^{15}$ Viscous fluxes are approximated with central differences.

Although a space marching code would normally be sufficient for describing flow on a flat plate it is not appropriate for the present study because identification of upstream influences of the surface temperature jump is of primary concern. Consequently, global relaxation of the governing equations is employed.

\section{Grid Refinement - Flat Plate}

Sequences of successively finer streamwise grids were used to resolve the flow in the vicinity of $x_{J}$. The initial grid used 200 cells from the leading to trailing edge. Subsequent grids picked up converged solutions 


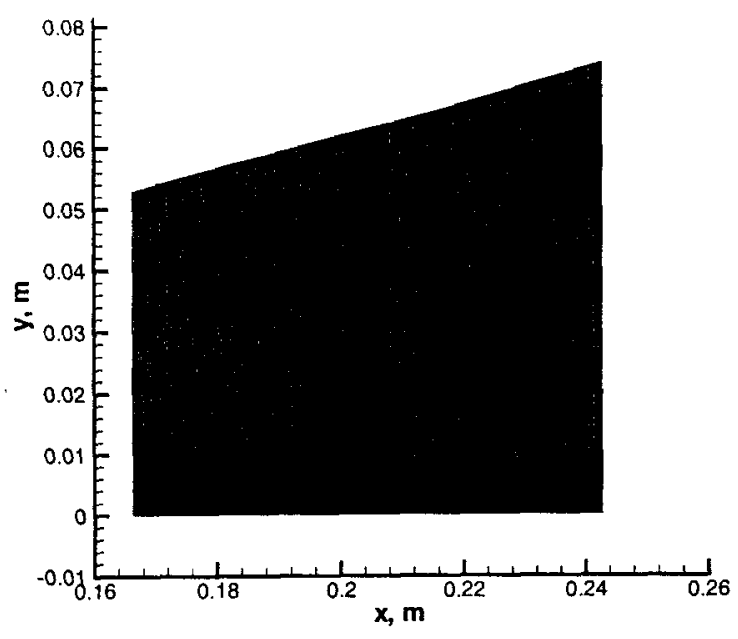

Fig. 3 Test case grid for simulation with $T_{w}=300$. $K$ and factor 100 increase in streamwise resolution around $x_{J}=20.455 \mathrm{~cm}$ in embedded block.

of the flow sufficiently upstream of $x_{J}$ to preclude any upstream effects of the jump on the inflow profiles. The simulations used 128 cells across the shock layer; comparisons to a 64 cell simulation indicates grid convergence. The outer boundary is aligned with the shock emanating from the leading edge. The cell Reynolds number at the wall averaged 0.25 . The maximum stretching factor in the normal direction was 1.12 occuring near the middle of the boundary layer at $\mathrm{k}=$ 50.

Streamwise resolution on the coarsest grid near $x_{J}$ is approximately $3 \mathrm{~mm}$. The next grid in sequence uses a single cell deep feeder block and a single, refined block (200 x 128) extracted from the coarser block to resolve upstream and downstream influence of the temperature jump at $x_{J}$. It has streamwise resolution near $x_{J}$ of approximately $0.3 \mathrm{~mm}$.

The finest grid uses a single cell deep feeder block and 3 active blocks ( $89 \times 128),(128 \times 128)$, and (90 $x$ 128) resolving the shock layer near $x_{J}$. The second of these active blocks provides another factor 10 increase in streamwise resolution near $x_{J}$ to approximately $0.03 \mathrm{~mm}$. An overview of the fine grid is shown in Fig. 3.

\section{Grid Refinement - RLV}

Streamwise grid refinement progressed in the same manner as the flat plate. The final computational domain includes a windside section of the RLV extending from $10 \mathrm{~m}$ to $23 \mathrm{~m}$ behind the nose. The final surface grid and a representative surface heating distribution are presented in Fig. 4. The inflow boundary is outside the influence of the temperature jump. The fine, streamwise mesh spacing across the jump is approximately $.008 \mathrm{~m}$ so that the local Reynolds number based on streamwise mesh spacing is approximately

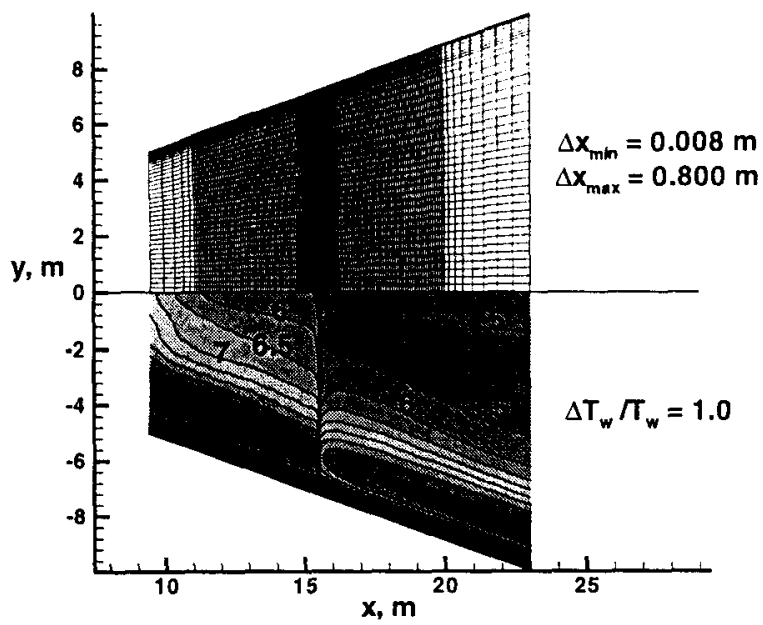

Fig. 4 Heating and surface mesh on RLV test case with $\Delta T_{w} / T_{w}=1.0$.

70. A corresponding value for Reynolds number based on streamwise mesh spacing on the flat plate is approximately 40 for the low Reynolds number tests.

The grid refinement process referenced an available surface grid of the RLV, not the original CAD description. Consequently, the grid refinement produces a faceted surface in regions where the curvature is nonzero in the streamwise direction. Faceting is most evident near the outboard chines when viewing surface pressure contours. Nevertheless, the nondimensionalization used herein subtracts out the faceted baseline reference results for pressure and heating.

\section{Results and Discussion}

\section{Pressure Field above the Surface}

Pressure contours across the boundary layer of the flat plate at $M_{\infty}=6$ and $R e_{L}=2.710^{6}$ are presented in Fig. 5. The constant wall temperature case is in Fig. $5 a$ and the temperature jump at $x_{J}=20.455$ $\mathrm{cm}$ is in Fig. 5b. Vertical lines in this figure indicate computational block boundaries of the fine grid region surrounding the discontinuity.

The background pressure field in Fig. 5a is nearly constant and a very weak oscillation across the boundary layer edge manifests itself as a jagged profile. If we take a cut across the boundary layer and weak shock emanating from the leading edge there is no indication of a pressure oscillation in Fig. 6a. However, in the highly expanded scale of Fig. $6 \mathrm{~b}$ an oscillation is visible at the boundary layer edge. Numerical experiments on grid stretching and limiters failed to reveal a numerical cause for this profile; we nute only that relative mimima and maxima follow streamlines along the boundary layer edge to the highly curved portion of the leading edge shock. Whatever their cause, the magnitude of the oscillations is two orders smaller than 


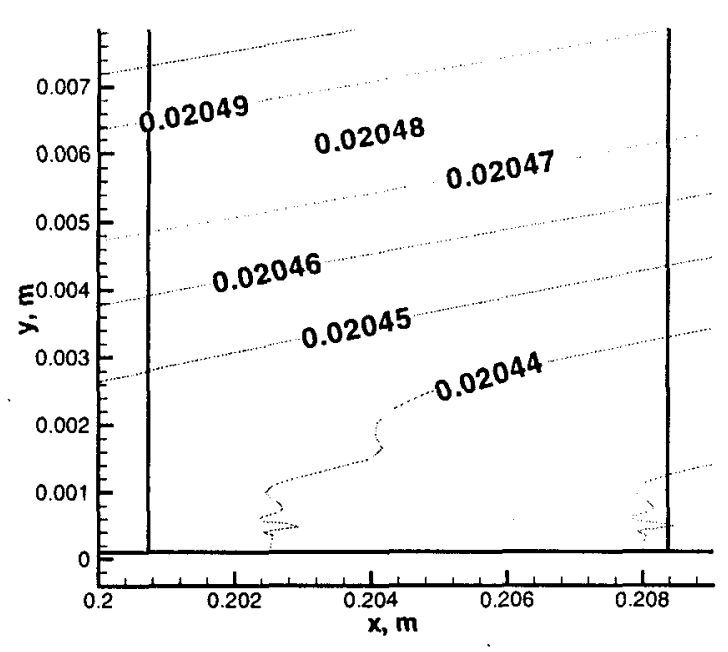

a) without temperature jump

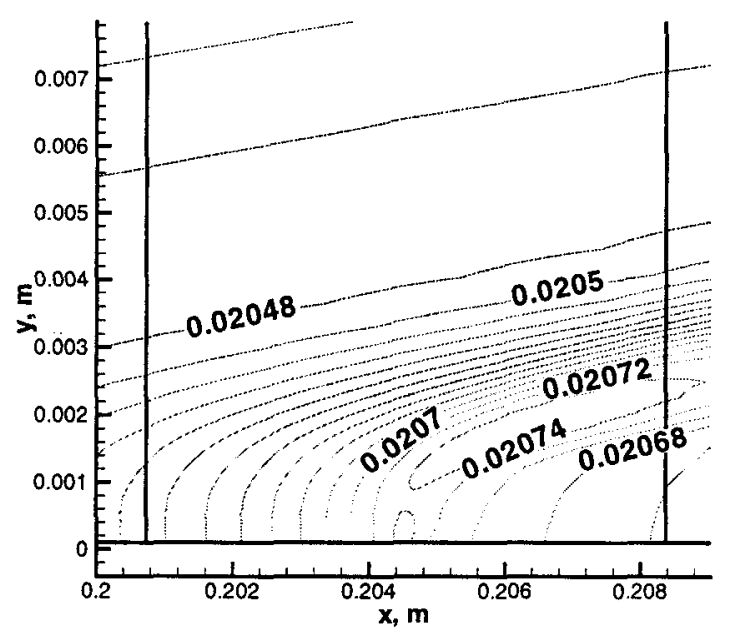

b) with temperature jump

Fig. 5 Comparison of pressure contours around $x_{J}=20.455 \mathrm{~cm}$ in embedded block with and without temperature jump.

the magnitude of pressure perturbations caused by the temperature jump.

The imposition of an abrupt $10 \%$ increase in wall temperature at $x_{J}$ induces changes in pressure and heating that are felt upstream and in the wake of the discontinuity. In Fig. 5b, the pressure jump is largest at $x_{J}$ and the perturbation moves up to the boundarylayer edge. Coalescing pressure waves followed by an expansion are observed trailing off to the right. The pressure oscillation from the simple, constant wall temperature boundary condition disappears, masked by the larger gradients in induced pressure.

Pressure contours in the symmetry plane across the RLV shock layer associated with temperature jumps

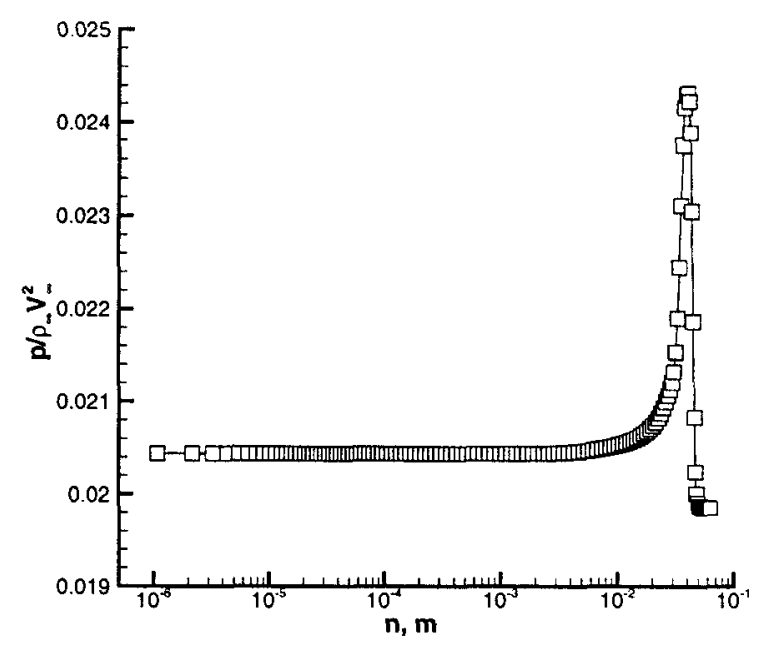

a) profile across shock layer

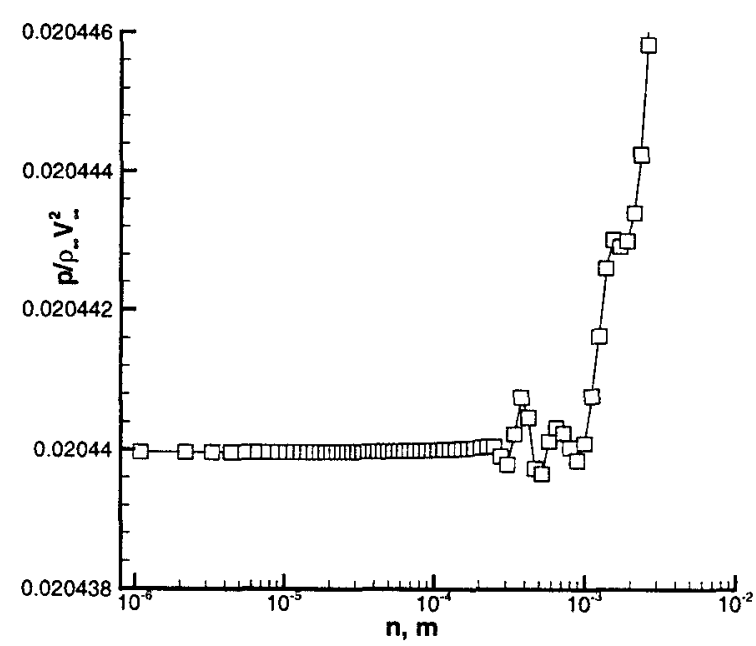

b) profile across boundary layer

Fig. 6 Pressure profles across layer. highlighting magnitude of oscillation near the boundary-layer edge.

$\Delta T_{w} / T_{w}$ equal to $0.0,0.1$, and 1.0 are presented in Fig. 7. The strengthening compression and expansion with increasing $\Delta T_{w}$ are evident in this figure. A reffected wave off the bow shock strikes the surface at $x=20$ $m$. for the highest temperature jump. The upstream influence of the discontinuity in all cases disappears within $.5 \mathrm{~m}$.

\section{Comparison of Triple-Deck Theory and CFD}

\section{Flat Plate}

A direct comparison of the present triple-deck theory with the Navier-Stokes numerical solution for the non-dimensional scaled pressure distribution $\hat{p}(\hat{x})$ is shown in Fig. 8 for the $M_{\infty}=2$, high Reynolds number case. A range of positive and negative temperature 


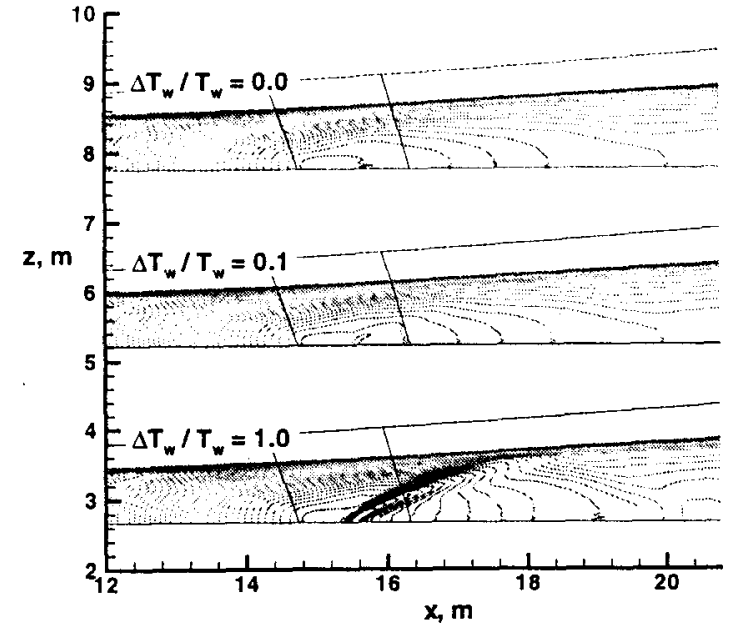

Fig. 7 Pressure contours in symmetry plane associated with different temperature jumps at $x_{J}$ for RLV test case.

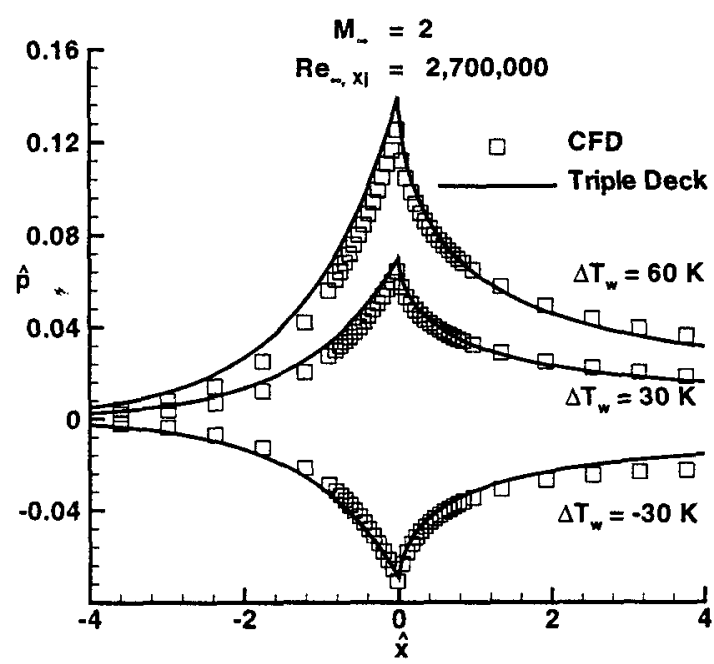

Fig. 8 Effect of temperature jump amplitude on interactive pressure distribution for $M_{\infty}=2$ and $R e_{L}=2,700,000$.

jumps are considered. It is seen that the shape, extent and magnitude of the pressure perturbation are all very accurately predicted by the triple-deck theory throughout both the upstream influence and downstream wake regions of the interaction. The same excellent comparisons are also obtained for the corresponding non-dimensional heat transfer predictions $\hat{q}$ as shown in Fig. 9. The theory is clearly able to properly capture the important sharp peaking of the local heating that occurs immediately downstream of the temperature jump. The slow subsequent approach in the wake toward the proper asymptotic value pertaining to the new wall temperature is also captured.

The application of the leading asymptotic approxi-

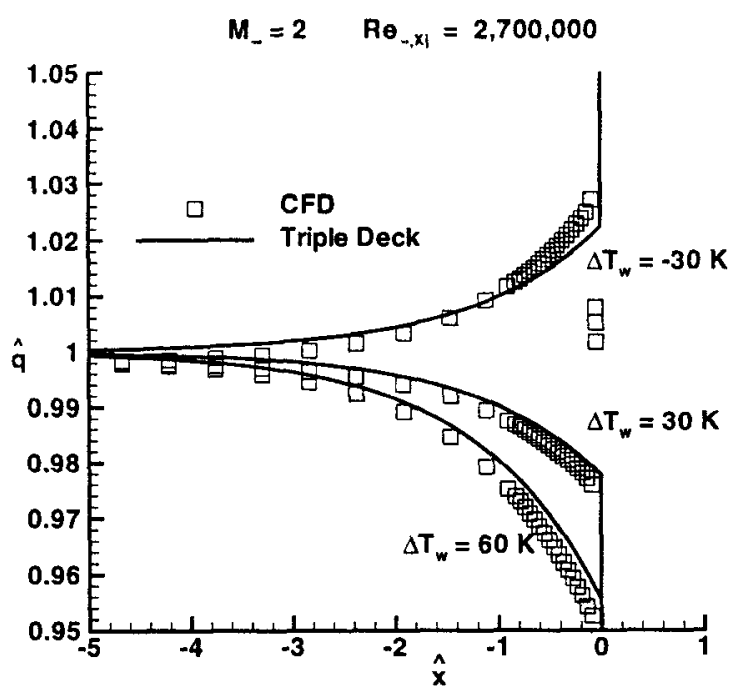

a) Upstream of Jump

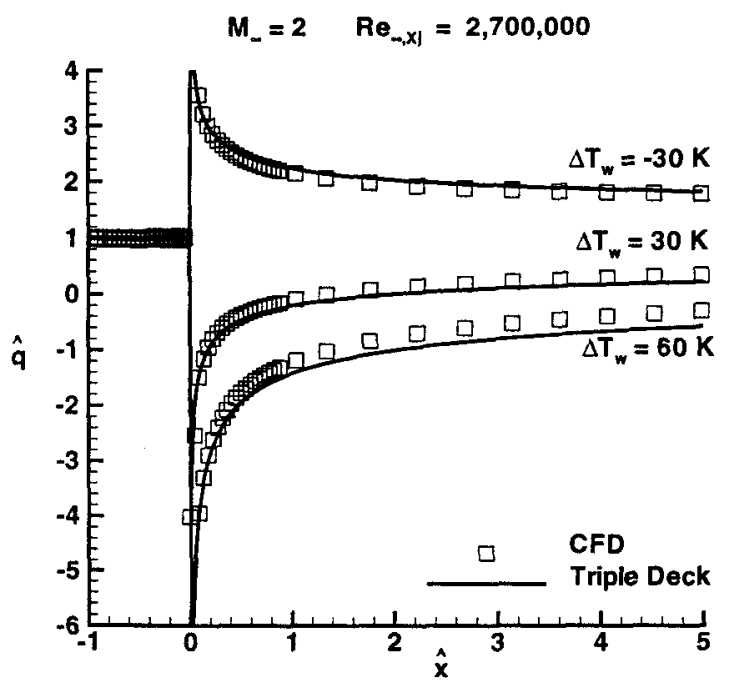

b) Downstream of Jump

Fig. 9 Effect of temperature jump amplitude on interactive heating distribution for $M_{\infty}=2$ and $R e_{L}=2,700,000$.

mation $(\epsilon \rightarrow 0)$ of triple-deck theory at finite Reynolds numbers introduces some error that may be assessed by comparing with the Navier-Stokes numerical solutions. This comparison is presented in Fig. 10, where the $\hat{p}(\hat{x})$ distributions for the $M_{\infty}=2$ test case with $\Delta T_{w} / T_{w}=0.1$ at two different Reynold numbers is compared to triple-deck theory. Note that all Reynolds numbers collapse to a single curve in the triple-deck theory, which is most accurate for $R e_{L} \rightarrow \infty$. It can be seen that the finite Reynolds number effect serves to slightly reduce the streamwise extent of the interaction in $\hat{x}$ primarily in the upstream influence region, the reduction increasing with decreasing $R e_{L}$. This effect stems from the fact that in contrast to what is 


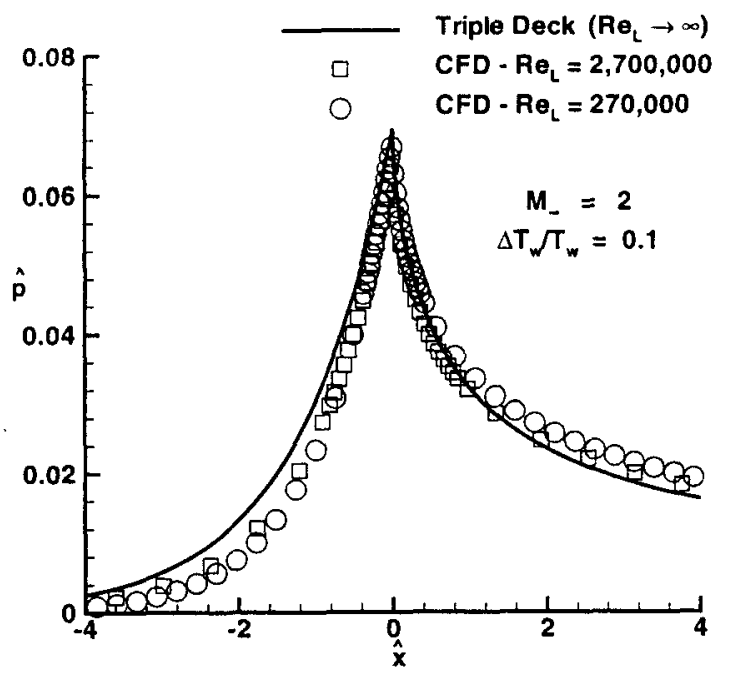

Fig. 10 Finite Reynolds number effect on interactive pressure field.

assumed in the triple-deck model, the upstream region of any real interaction depends on conditions at the upstream start of the interaction rather than at $\mathrm{X}=\mathrm{L}$; as discussed by Stewartson, this leads to a modest Reynolds number effect that reduces the effective upstream influence distance in $\hat{x}$. The present results for the temperature jump problem are also in qualitative agreement with an earlier theoretical study ${ }^{16}$ of finite Reynolds number effects on oblique shockgenerated interactions.

Turning to a consideration of the Mach number effect, Fig. 11 presents a comparison of the theoretical $\hat{p}(\hat{x})$ distribution (which is independent of $M_{\infty}$ ) with the CFD solutions obtained at Mach numbers of 2, 4, and 6. It is seen that as the freestream Mach number increases and enters the hypersonic regime, the numerical simulation of the exact equations predicts a progressively more significant reduction in upstream influence along with a modest reduction in the corresponding maximum pressure. Analysis indicates that this Mach number effect is in fact the combined result of nonlinear effects in both the inviscid pressure-flow deflection relationship and the temperature jump interaction (neither of which are accounted for in the present linearized treatment). Interestingly there is little attendant Mach number effect on the downstream wake behavior.

The corresponding effects of Mach number on heating across the temperature jump are shown in Fig. 12. As Mach number increases the upstream influence of the temperature jump on heating decreases in $\hat{x}$ coordinates faster than predicted by theory. This trend is the same as observed in Fig. 11 for the upstream influence of Mach number to $\hat{p}(\hat{x})$. A magnified view of the downstream asymptotic behavior of $\hat{q}(\hat{x})$ as a function of Mach number is shown in Fig. 12b. The down-

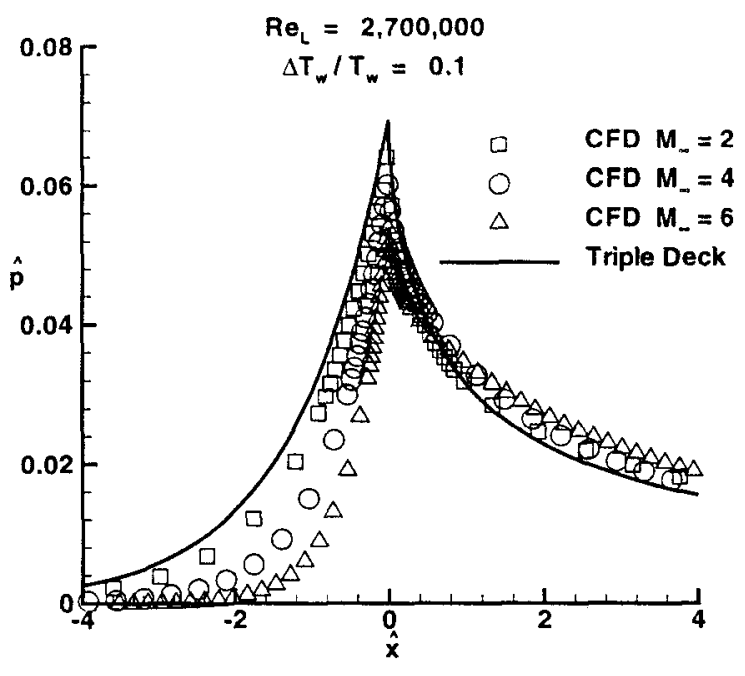

Fig. 11 Mach number effect on interactive pressure field.

stream behavior of $\hat{q}(\hat{x})$ is not independent of $M_{\infty}$ in the triple-deck theory because of the functional dependence of $C_{5}$ on $\beta$ and $C_{R E F}$. All three Mach number simulations show nearly a $20 \%$ difference in $\hat{q}$ at the most downstream $\hat{x}$ location. This trend will be evident in the RLV simulations as well. The magnitude of the parameter $C_{5}$ has the most influence on this comparison in this range where $\epsilon \hat{x}^{1 / 3}$ is of order 1 . Note that the limit for $\hat{q}(\hat{x})$ as $\hat{x} \rightarrow \infty$ is correct in Eq. 10 .

\section{$R L V$}

The RLV test case is intended to show how unmodified triple-deck theory performs in a regime that is of practical interest to the thermal protection system design community. The RLV simulation includes effects of chemical nonequilibrium ( 5 species air model) and a fully catalytic wall boundary condition. The limiting form of Eq. 10 does not account for species diffusion to the surface. The results obtained here will be used to guide more comprehensive analytic modeling of both temperature and catalytic jump problems in the context of triple-deck theory. In spite of these caveats, the theory captures much of the interaction quite well.

The strong three-dimensionality of the flow is evident in the surface heating contours of Figs. 4 and 13. The extent of the interaction can be discerned by comparing the heating rates for $\Delta T_{w} / T_{w}$ equal to 0 and 0.1 in Fig. 13. It is also clear that this perturbation in temperature is very low relative to the adiabatic wall temperature for this case - thus evolving an extended study with a larger perturbation $\Delta T_{w} / T_{w}=1.0$.

A comparison of triple-deck theory and numerical simulation for the pressure and heating across the temperature jump $\Delta T_{w} / T_{w}=0.1$ is presented in Fig. 14 for the centerline location. The agreement in upstream influence and downstream asymptotic behavior of pressure is very good. Only the peak level in pres- 


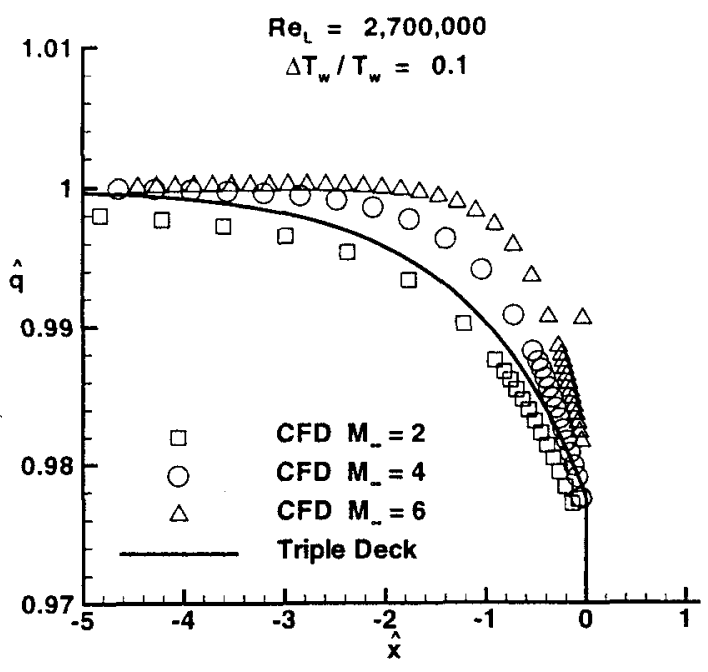

a) Upstream of Jump

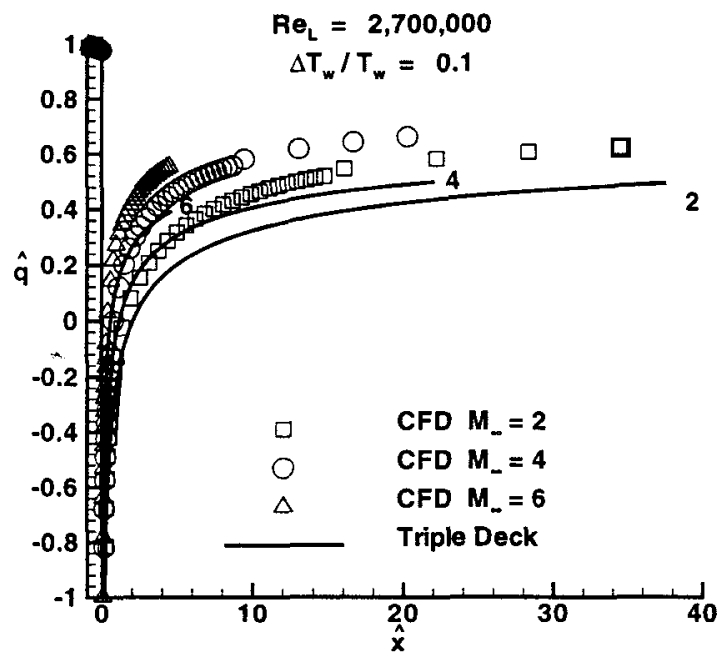

b) Downstream of Jump

Fig. 12 Mach number effect on interactive heating distribution for $\Delta T_{w} / T_{w}=0.1$ and $R e_{L}=2,700,000$ on expanded $\hat{x}$ scale.

sure disturbance at the jump is overpredicted, possibly because the theory does not account for the varying heat capacity across the boundary layer. The upstream heating influence is quite similar to the agreement found at Mach 2 for the flat plate. The relaxation to the asymptotic value of heating downstream of the jump appears offset - though in magnitude there is less than a $2 \%$ decrement in the asymptotic heating rate associated with the $10 \%$ increase in surface temperature.

A comparison of triple-deck theory and numerical simulation for the pressure and heating across the temperature jump $\Delta T_{w} / T_{w}=0.1$ is presented in Fig. 15 for the outboard location. The flow in this re-

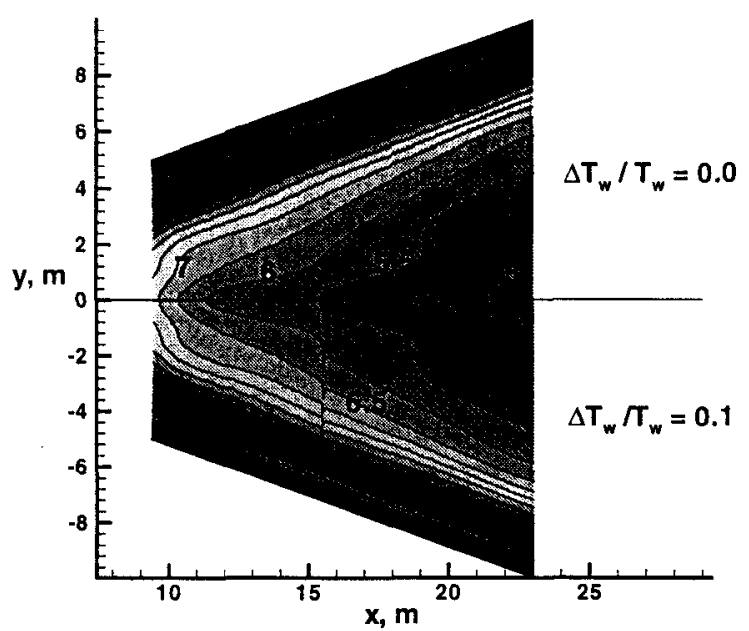

Fig. 13 Surface heating rates for RLV test case with and without temperature jump, $\Delta T_{w} / T_{w}=0.1$.

gion is more strongly three-dimensional and the local Reynolds number is a factor 2.5 smaller than at the centerline. The comparison follows the same trends as at the centerline but the magnitude of differences is larger.

A comparison of triple-deck theory and numerical simulation for the pressure and heating across the larger temperature jump $\Delta T_{w} / T_{w}=1.0$ is presented in Fig. 16 for the centerline location. As noted previously, this temperature perturbation can no longer be considered small in the context of the triple-deck formulation. Nevertheless, it is small relative to the adiabatic wall temperature. The pressure distribution scales exactly with $\Delta T_{w} / T_{w}$ - the corresponding curves for theory and CFD in Fig. 14 are almost identical to those in Fig. 16. The CFD heating distribution is approximately $10 \%$ higher than theory at $\hat{x}=20$, a location near the downstream edge of the fine grid in Fig. 4. The CFD heating distribution is approximately $6 \%$ higher than theory at $\hat{x}=200$, a location near the outflow boundary of the larger domain.

In all cases, including the flat plate, the CFD simulation approaches its asymptotic level faster than predicted by triple deck theory in the range $1<\hat{x}<$ $\operatorname{Order}(100)$. The behavior of $\hat{q}$ in this domain is dominated by the term $\frac{C_{5}}{\epsilon \tilde{x}^{1 / 3}}$ in Eq. 10 which is of $\operatorname{Order}(1)$ in this range.

\section{Concluding Remarks}

This paper has presented a new analytical theory of wall temperature jump-generated interactions in supersonic flow that captures all the major physics of the resulting pressure and local heat transfer behavior, including upstream influence. The long range goal of this work is to provide analytic tools coupled with computational data sets to be used in the design of 


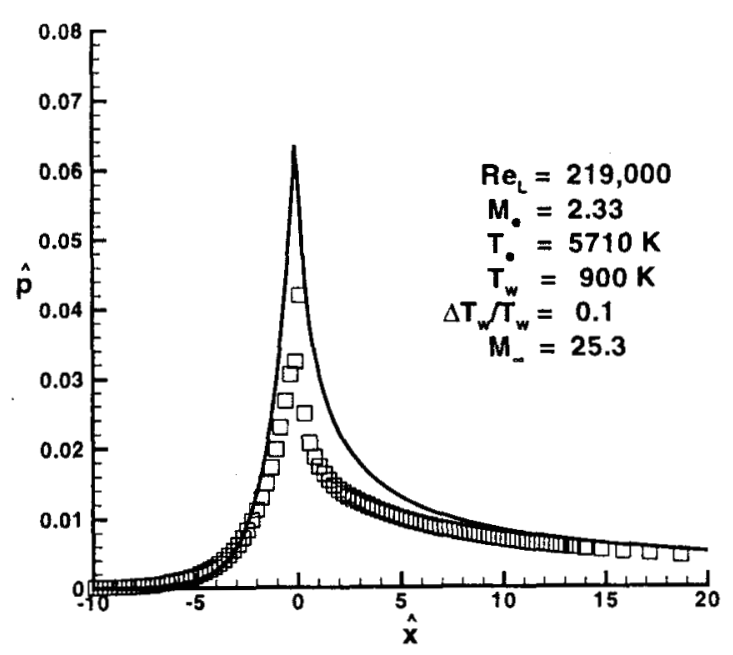

a) pressure

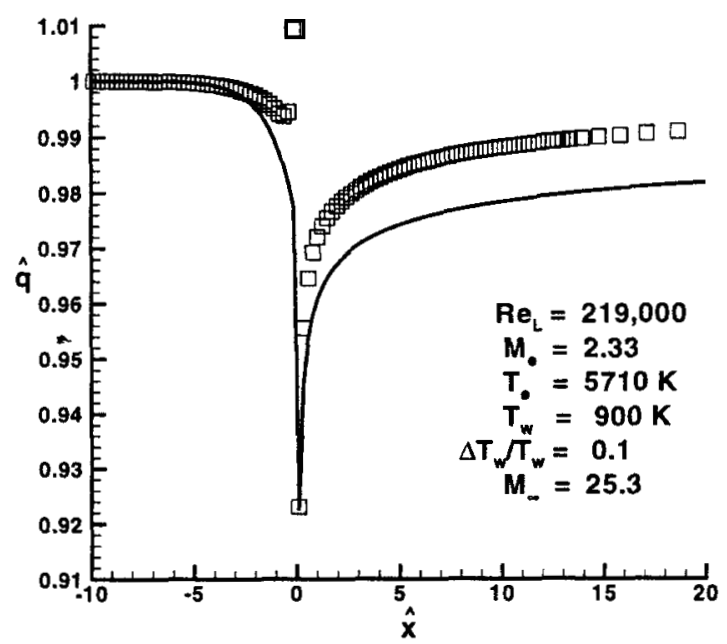

b) heating

Fig. 14 Triple-deck theory applied to RLV test case with $\Delta T_{w} / T_{w}=0.1$ on the windside plane of symmetry.

hypersonic vehicles in an Intelligent Synthesis Environment (ISE). Given a single, baseline CFD solution, the analytic tools will allow a designer to investigate effects of continuous global changes or discontinuous local changes to thermal protection system surface properties on heating.

Comparisons of triple-deck theory with numerical solutions of the exact Navier-Stokes equations for a perfect gas on a flat plate are presented. Good agreement establishes confidence that the closed form analytical results are properly formulated and coded. In the case of laminar, high Reynolds number, supersonic flow $\left(M_{\infty}=2\right.$ and $\left.R e_{L}=2.710^{6}\right)$ theoretical predictions for the upstream and downstream behavior

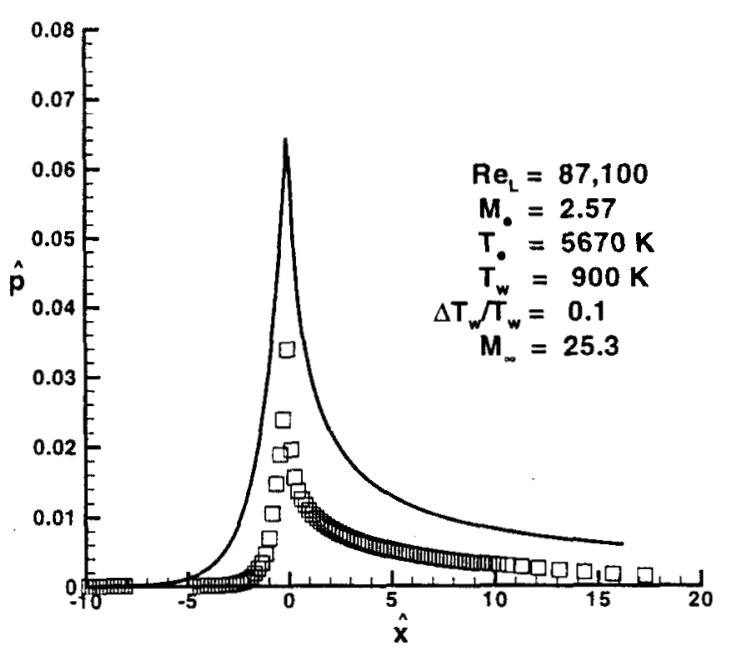

a) pressure

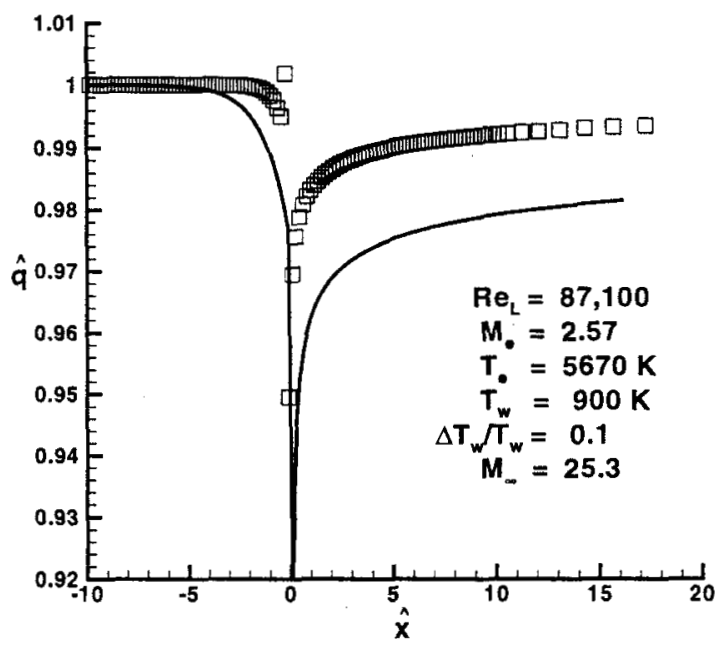

b) heating

Fig. 15 Triple-deck theory applied to RLV test case with $\Delta T_{w} / T_{w}=0.1$ near the outboard location.

of surface pressure and heating in the vicinity of the discontinuity are in excellent agreement with computational simulation. Some decrement of theory is noted for larger edge Mach numbers (hypersonic) and for the asymptotic behavior of heating far downstream from the jump.

A Reusable Launch Vehicle (RLV) test case with an imposed surface temperature discontinuity is intended to show how unmodified triple-deck theory performs in a regime that is of practical interest to the thermal protection system design community. The RLV simulation includes effects of chemical nonequilibrium (5 species air model) and a fully catalytic wall boundary condition. Although the nonequilibrium physics of this problem is not included in the present triple-deck for- 


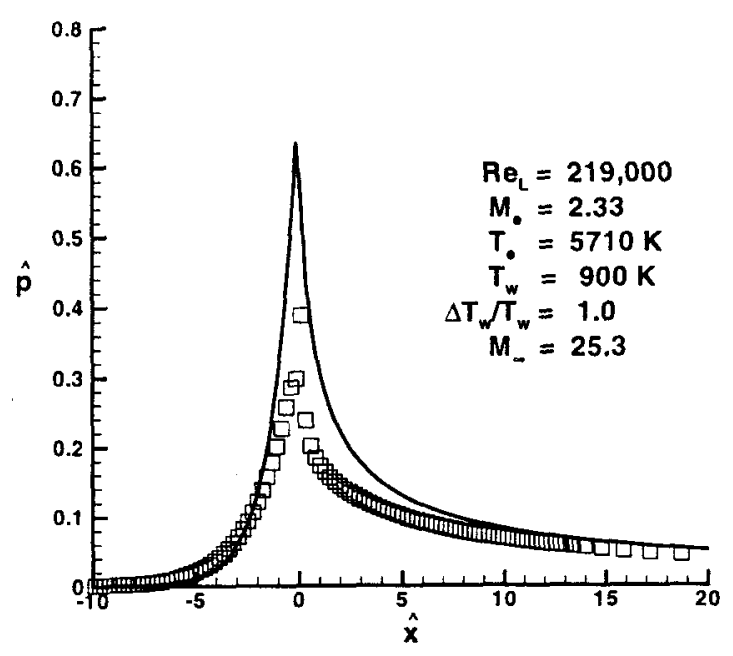

a) pressure

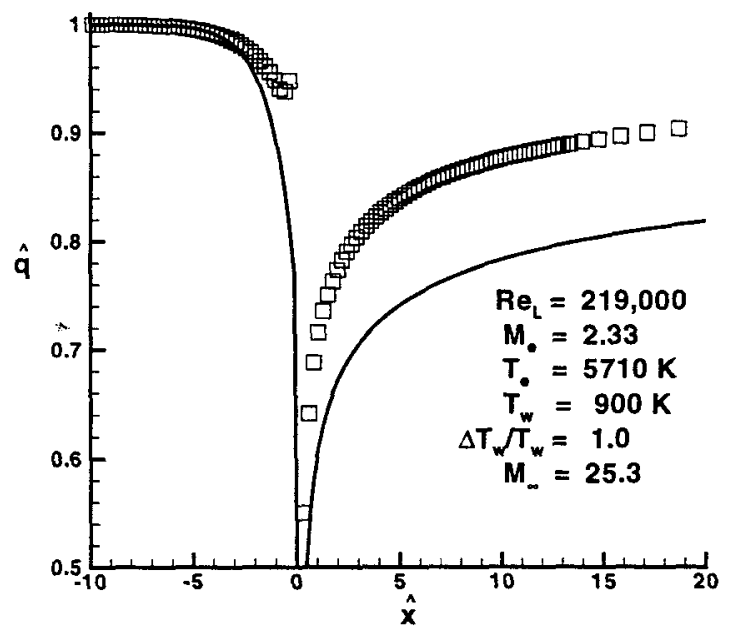

b) heating

Fig. 16 Triple-deck theory applied to RLV test case with $\Delta T_{w} / T_{w}=1.0$ on the windside plane of symmetry.

mulation, the theory captures much of the interaction quite well. Guidance for adapting a strongly threedimensional, CFD flow simulation (on a grid that is too coarse to resolve the interactions across the discontinuity) into the context of a triple-deck analysis is provided.

A problem related to the temperature jump is that of dissociated gas flow past a wall with a jump in the surface catalycity (heterogeneous recombination rate). This situation arises in connection with thermal protection system (TPS) design for hypervelocity vehicles operating in the high-altitude nonequilibrium flow chemistry regime where there is a discontinuity in the surface material at some station along the body.
Application of triple-deck theory to this more complex problem will be guided by the results obtained herein for the RLV application.

\section{References}

'Inger, G. R. and Gnoffo, P. A., "Analytical and Computational Study of Wall Temperature Jumps in Supersonic Flow," AIAA 99-0226, AIAA, January 1999.

${ }^{2}$ Inger, G. R. and Gnoffo, P. A., "Hypersonic Entry Heating with Discontinuous Surface Catalycity: A Combined Analytic/CFD Approach," AIAA Paper 96-2150, June 1996.

${ }^{3}$ Gnoffo, P. A. and Inger, G. R., "Analytic Corrections to Computational Heating Predictions Accounting for Changes in Surface Catalysis," Journal of Spacecraft and Rockets, Vol. 35, No. 4, July-August 1998, pp. 417-423.

${ }^{4}$ Gnoffo, P. A. and Inger, G. R., "Analytic Corrections to CFD Heating Predictions Accounting for Changes in Surface Catalysis," AIAA 96-1800, AIAA, June 1996.

${ }^{5}$ Gnoffo, P. A., "Point-Implicit Relaxation Strategies for Viscous, Hypersonic Flows," Computational Methods in Hypersonic Aerodynamics, edited by T. K. S. Murthy, Computational Mechanics Publications, Kluwer Academic Publishers, 1991, pp. 115-151.

${ }^{6}$ Gnoffo, P. A., "Upwind-Biased, Point-Implicit Relaxation Strategies for Viscous, Hypersonic Flows," AIAA 89-1972CP, AIAA, June 1989.

${ }^{7}$ Gnoffo, P. A., "An Upwind-Biased, Point-Implicit Relaxation Algorithm for Viscous, Compressible Perfect-Gas Flows," TP 2953, NASA, February 1990.

${ }^{8}$ Gnoffo, P. A., "Code Calibration Program in Support of the Aeroassist Flight Experiment," Journal of Spacecraft and Rockets, Vol. 27, No. 2, March-April 1990, pp. 131-142.

${ }^{9}$ Weilmuenster, K. J. and Gnoffo, P. A., "Aeroassisted Flight Experiment Aerodynamic Characteristics at Flight Conditions," Journal of Spacecraft and Rockets, Vol. 27, No. 6, NovemberDecember 1990, pp. 684-686.

${ }^{10}$ Thompson, R. A. and Gnoffo, P. A., "Application of the LAURA Code for Slender Vehicle Aerothermodynamics," Journal of Spacecraft and Rockets, Vol. 29, No. 1, January-February 1992, pp. 16-23.

${ }^{11}$ Weilmuenster, K. J., Gnoffo, P. A., and Greene, F. A., "Navier-Stokes Simulations of Orbiter Aerodynamic Characteristics Including Pitch Trim and Bodyflap," Journal of Spacecraft and Rockets, Vol. 31, No. 3, May-June 1994, pp. 355-366.

${ }^{12}$ Gnoffo, P. A., Weilmuenster, K. J., and Alter, S. J., "Multiblock Analysis for Shuttle Orbiter Re-entry Heating from Mach 24 to Mach 12," Journal of Spacecraft and Rockets; Vol. 31, No. 3, May-June 1994, pp. 367-377.

${ }^{13}$ Roe, P. L., "Approximate Riemann Solvers, Parameter Vectors, and Difference Schemes," Journal of Computational Physics, Vol. 43, October 1981, pp. 357-372.

${ }^{14}$ Harten, A., "High Resolution Schemes for Hyperbolic Conservation Laws," Journal of Computational Physics, Vol. 49, No. 2, February 1983, pp. 357-393.

${ }^{15}$ Yee, H. C., "On Symmetric and Upwind TVD Schemes," T.M 88325, NASA, June 1986.

${ }^{16}$ Hussaini, M. Y., Baldwin, B. S., and McCormack, R. L., "Asymptotic Features of Shock Wave - Boundary Layer Interaction," AIAA Journal, Vol. 18, No. 8, August 1980, pp. 10141016. 